\title{
LAS ADAPTACIONES CURRICULARES DE PEQUEÑO PORTE O NO SIGNIFICATIVAS EN EL CONTEXTO BRASILEÑO
}

\author{
Eladio Sebastián Heredero ${ }^{1}$
}

\begin{abstract}
RESUMEN
La presente comunicación pretende conceptualizar qué se entiende por adaptaciones curriculares de pequeño porte dentro de la legislación brasileña, organizar todas las respuestas que a diferentes niveles pueden darse dentro de la escuela inclusiva que en estos momentos vivimos y además aportar ideas y estrategias concretas para organizar esta medida de atención a la diversidad de la manera más eficaz posible. A partir de la reflexión sobre el trabajo en el aula y de la experiencia vivida en contextos escolares brasileños 2 sobre la base de una metodología de investigación acción se hacen las aportaciones técnicas para su desarrollo en los centros educativos y se dan estrategias concretas para su organización en las instituciones escolares. Por último, se pretende aportar un documento denominado DACI en el cual se recojan todas las medidas que para un determinado alumno o grupo se quieran hacer a fin de ajustar la respuesta educativa a las necesidades concretas por él planteadas en el contexto del aula y de la escuela.
\end{abstract}

Palabras-clave: Adaptación curricular, necesidades educativas, escuela inclusiva, atención a la diversidad, respuestas educativas, metodología.

\section{INTRODUCCIÓN}

La Ley de Directrices y Bases, de Educación Brasileña, Ley 9394 de 20 de diciembre de 1996 señala concretamente sobre este tema:

\section{CAPÍTULO V DA EDUCAÇÃO ESPECIAL:}

Art. 59. Os sistemas de ensino assegurarão aos educandos com necessidades especiais: I - currículos, métodos, técnicas, recursos educativos e organização específicos, para atender às suas necessidades;

II - terminalidade específica para aqueles que não puderem atingir o nível exigido para a conclusão do ensino fundamental, em virtude de suas deficiências, e aceleração para

\footnotetext{
1 Profesor de la Universidad de Alcalá. Departamento de Didáctica. Área Didáctica y Organización Escolar. Guadalajara (España). eladio.sebastian@uah.es

2 El autor lleva impartiendo diferentes cursos de formación del profesorado brasileño en materia de atención a la diversidad y adaptación del currículo y haciendo el seguimiento de la inclusión de alumnos con necesidades educativas especiales en el aula ordinaria desde 1999.
} 
concluir em menor tempo o programa escolar para os superdotados;

III - professores com especialização adequada em nível médio ou superior, para atendimento especializado, bem como professores do ensino regular capacitados para a integração desses educandos nas classes comuns;

IV - educação especial para o trabalho, visando a sua efetiva integração na vida em sociedade, inclusive condições adequadas para os que não revelarem capacidade de inserção no trabalho competitivo, mediante articulação com os órgãos oficiais afins, bem como para aqueles que apresentam uma habilidade superior nas áreas artística, intelectual ou psicomotora;

$V$ - acesso igualitário aos beneficios dos programas sociais suplementares disponíveis para orespectivo nível do ensino regular.

Art. 60. Os órgãos normativos dos sistemas de ensino estabelecerão critérios de caracterização das instituições privadas sem fins lucrativos, especializadas e com atuação exclusiva em educação especial, para fins de apoio técnico e financeiro pelo Poder Público.

Parágrafo único. O Poder Público adotará, como alternativa preferencial, a ampliação do atendimento aos educandos com necessidades especiais na própria rede pública regular de ensino, independentemente do apoio às instituições previstas neste artigo.

Para llegar a estos currículos específicos o adaptaciones es posible establecer una secuencia teórica de medidas. No obstante, habrá que ser lo suficientemente flexibles para considerar, tanto las necesidades reales de los estudiantes como las posibilidades organizativas y funcionales de las instituciones educativas dentro del modelo de escuela inclusiva.

Adaptaciones de pequeño porte, ordinarias o no significativas

Programas de refuerzo educativo y apoyo

Repetición de curso

Adaptaciones de gran porte, extraordinarias o significativas

\section{Concepto de adaptación curricular}

Las adaptaciones se conciben como un instrumento que puede y debe tratar de lograr el avance en el desarrollo general de cada persona.

“(...) a Secretaria de Educação Fundamental e a Secretaria de Educação Especial, em ação conjunta, produziram o material didático-pedagógico intitulado 'Adaptações 
Curriculares', que compõem o conjunto dos Parâmetros Curriculares Nacionais-PCN, inserindo na concepção da escola integradora defendida pelo Ministério da Educação. Nesse sentido, a adequação curricular ora proposta procura subsidiar a prática docente, propondo alterações a serem desencadeadas na definição dos objetivos, no tratamento e desenvolvimento dos conteúdos, no transcorrer de todo processo avaliativo, na temporalidade e na organização do trabalho didático-pedagógico no intuito de favorecer a aprendizagem do aluno” (MEC, 1999).

En sentido amplio, el marco de referencia de las mismas es, pues, el currículo ordinario: "Las adaptaciones curriculares suponen una estrategia didáctica dirigida a facilitar, en la medida de lo posible, que los alumnos con dificultades se enfrenten en las mejores condiciones al aprendizaje del área".

En sentido más restringido son: "Son el conjunto de modificaciones que se realizan en los objetivos, contenidos, criterios y procedimientos de evaluación, actividades y metodología para atender a las diferencias individuales de los alumnos."

No documento Adaptações curriculares: estratégias para a educação de alunos com necessidades educacionais especiais, se diz:

“O projeto pedagógico da escola, como ponto de referência para definir a prática escolar, deve orientar a operacionalização do currículo, como um recurso para promover o desenvolvimento e a aprendizagem dos alunos, considerando-se os seguintes aspectos:

- a atitude favorável da escola para diversificar e flexibilizar o processo de ensinoaprendizagem, de modo a atender às diferenças individuais dos alunos;

- a identificação das necessidades educacionais especiais para justificar a priorização de recursos e meios favoráveis à sua educação;

- a adoção de currículos abertos e propostas curriculares diversificadas, em lugar de uma concepção uniforme e homogeneizadora de currículo;

- a flexibilidade quanto à organização e ao funcionamento da escola, para atender à demanda diversificada dos alunos;

- a possibilidade de incluir professores especializados, serviços de apoio e outros, não convencionais, para favorecer o processo educacional.

Essa concepção coloca em destaque a adequação curricular como um elemento dinâmico da educação para todos e a sua viabilização para os alunos com necessidades 
educacionais especiais: não se fixar no que de especial possa ter a educação dos alunos, mas flexibilizar a prática educacional para atender a todos e propiciar seu progresso em função de suas possibilidades e diferenças individuais.

Pensar em adequação curricular, significa considerar o cotidiano das escolas, levandose em conta as necessidades e capacidades dos seus alunos e os valores que orientam a prática pedagógica. Para os alunos que apresentam necessidades educacionais especiais essas questões têm um significado particularmente importante."

Estas adaptaciones del currículo pueden ser de dos tipos en función del grado de modificación de los elementos del mismo y de la respuesta a las necesidades educativas planteadas por el alumnado, a saber: Adaptaciones curriculares extraordinarias, significativas o de gran porte y Adaptaciones curriculares ordinarias, no significativas o de pequeño porte. En este caso sólo nos vamos a referir a estas últimas.

\section{Adaptaciones curriculares ordinarias, no significativas o de pequeño porte:}

Son modificaciones que se realizan en los diferentes elementos de la programación diseñada para todos los alumnos de un aula o ciclo para responder a las diferencias individuales, pero que no afectan prácticamente a las enseñanzas básicas del currículo oficial ya que no hay modificación sustancial de los elementos básicos del mismo

Puede precisarlas cualquier alumno que tenga necesidades educativas de carácter temporal, y por supuesto se pueden proponer para alumnos con NEE como medidas previas o complementarias. Precisamente, el profesor las realiza para que los alumnos puedan conseguir las capacidades expresadas en el currículo oficial. Son la estrategia fundamental para conseguir la individualización de la enseñanza y constituyen las acciones que debería realizar todo profesor en el ejercicio responsable de su acción como profesor responsable de clase.

- Afectan parcialmente a los elementos del currículo o a los elementos de acceso: espacios, tiempos, agrupamientos, materiales, recursos... en general, suponen leves alteraciones y afectan con frecuencia a la metodología didáctica (ritmo de instrucción, secuenciación y planificación de contenidos y tareas) ...

- No afectan a los elementos básicos del currículo del área o materia. Se trata de ajustes poco significativos en los contenidos y objetivos, formas de enseñar, procedimientos de evaluación o recursos utilizados. En este sentido, aunque se tratan de adecuar a las necesidades del alumno, no suponen una extremada individualización de la 
enseñanza. Esto hace que puedan establecerse líneas generales o adaptaciones tipo que son factibles de utilizar con un gran número de alumnos con características similares.

\section{Orientaciones didácticas para las adaptaciones curriculares de pequeño porte.}

Pueden consistir en:

- Adecuar los objetivos, contenidos o criterios de evaluación.

- Priorizar determinados objetivos, contenidos o criterios de evaluación.

- Cambiar la temporalización de los objetivos o criterios de evaluación.

- Introducir contenidos, objetivos o criterios de evaluación.

- Todos los cambios necesarios en metodología.

Mantiene los objetivos y contenidos propios del ciclo: qué enseñar y evaluar. La programación ordinaria sufre pequeñas modificaciones, afectando al cómo y cuándo enseñar y evaluar. Por lo tanto, repercutirá en la organización del aula, en la secuenciación de los contenidos, en la metodología general y específica si así fuese preciso, en la creación y desarrollo de actividades, en los sistemas de evaluación...etc.

Las orientaciones didácticas en las adaptaciones curriculares de pequeño porte o no significativas han de tener en cuenta:

a) Su aplicación y desarrollo corresponde a los profesores de área o materia, bajo la coordinación del profesor responsable de clase. En ocasiones, si contemplan la utilización de recursos y materiales extras o bien apoyos puntuales, podrán contar con la colaboración de otros profesionales del centro educativo.

Serían equiparables en ciertos aspectos a los del refuerzo educativo sin necesidad de otros trámites que superen el nivel del centro escolar.

Parece oportuno que las adaptaciones curriculares ordinarias se apliquen según un orden que, aproximadamente, podría ser el siguiente:

b) Se debe comenzar por realizar cambios en:

- Los procedimientos de evaluación (pruebas orales, escritas, observación, cuaderno...)

- La organización o disposición física de la clase (en U, V, en círculo...), así como en el uso de otros espacios (biblioteca, audiovisuales, entorno del centro...)

- La temporalización (dedicar más tiempo a un contenido, facilitar tiempo extra en un 
examen...)

- Los agrupamientos (trabajo individual, pequeño grupo, por parejas, grupos flexibles).

- La metodología didáctica (presentación de contenidos, exposición del profesor, trabajo de los alumnos...), así como la realización de actividades alternativas (con distinto nivel de profundidad) o complementarias (para afianzar contenidos no dominados)

- El uso de materiales (recursos extras, fotocopias...)

Como este tipo de adaptaciones no afectan propiamente a los contenidos del área, sino a la forma en que se accede a los mismos (cómo y cuándo se enseña y evalúa) se deben proponer: - La selección de contenidos:

- Adecuados a los intereses y características de los alumnos.

- Necesarios para su desenvolvimiento en el mundo laboral.

- Realistas, puesto que los podrá contextualizar de la forma más inmediata y próxima posible en su entorno.

- El diseño de las actividades de enseñanza-aprendizaje:

- Partiendo de los conocimientos previos de cada alumno.

- Generando actividades de diversos tipos, tal como se explicitan en el currículo general.

- Propiciando la reflexión sobre el proceso de enseñanza por un lado y por otro sobre el proceso de aprendizaje, así como la interacción entre ambos.

- Elaborando conclusiones, que faciliten el avance y profundización.

Si las anteriores no surten efecto, procederá efectuar cambios en:

- Los contenidos (priorización, modificación o incorporación de otros nuevos).

- Los objetivos (priorización, modificación o incorporación de otros nuevos).

- Los criterios de evaluación (priorización, modificación o incorporación de otros nuevos).

Debe entenderse que estos cambios, que se refieren más al currículo del área (tomando este término en su acepción más restringida esto es: qué se enseña y evalúa), no deben afectar en ningún caso a la eliminación de los objetivos, contenidos y criterios de evaluación considerados básicos o mínimos.

En tanto que afecta a elementos básicos de las áreas, comprometen la superación de los objetivos de etapa. Ello hace que sólo se deban adoptar después de haber sopesado cuidadosamente todas las circunstancias que rodean al alumno y de haber fracasado otras medidas previas.

Por regla general, se propondrán para alumnos con dificultades de aprendizaje, habitualmente 
debidas a condiciones de déficit personal o social. En ocasiones, podrán aconsejarse para un alumno que no haya repetido curso, si no se prevé que esta última medida le permita recuperar los contenidos no superados. Igualmente, serán pertinentes cuando no se espera que el alumno obtenga el logro de los objetivos generales de la educación fundamental y por tanto su titulación, como medidas complementarias de adaptación curricular significativa o de gran porte.

No documento Adaptações curriculares: estratégias para a educação de alunos com necessidades educacionais especiais, se diz:

“Adaptações Não Significativas do Currículo

Organizativas:

- $\quad$ organização de agrupamentos

- $\quad$ organização didática

- $\quad$ organização do espaço

Relativas aos objetivos e conteúdos:

- $\quad$ priorização de áreas ou unidades de conteúdos

- $\quad$ priorização de tipos de conteúdos

- $\quad$ priorização de objetivos

- $\quad$ seqüenciação

- $\quad$ eliminação de conteúdos secundários

Avaliativas:

- $\quad$ adaptação de técnicas e instrumentos

- $\quad$ modificação de técnicas e instrumentos

Nos procedimentos didáticos e nas atividades:

- $\quad$ modificação de procedimentos

- $\quad$ introdução de atividades alternativas às previstas

- $\quad$ introdução de atividades complementares às previstas

- $\quad$ modificação do nível de complexidade das atividades

- $\quad$ eliminando componentes

- $\quad$ seqüenciando a tarefa

- $\quad$ facilitando planos de ação

- $\quad$ adaptação dos materiais

- $\quad$ modificação da seleção dos materiais previstos 
Na temporalidade:

- $\quad$ modificação da temporalidade para determinados objetivos e conteúdos

- previstos."

c) Aunque no es imprescindible se recomienda una evaluación diagnóstica previa que nos aporte la mayor información posible del alumno y la confección de un documento (DACI documento de adaptación curricular individual) en el que se concreten las adaptaciones propuestas. Este documento deberá incorporarse al expediente histórico del alumno.

\section{Criterios para realizar el DACI documento de adaptaciones curriculares individuales}

Trataremos de sintetizar la filosofía en los siguientes puntos:

- Partir siempre del currículo ordinario (proyectos politico-pedagógicos y programaciones didácticas de ciclo y área).

- Asegurarse de que hemos puesto en marcha otros recursos ordinarios (apoyo, refuerzo,...)

- Tener en cuenta los aspectos positivos que más destaquen del alumno.

- Buscar la posibilidad de logro de éxitos por parte del alumno y una motivación en sus propias producciones.

- Buscar que el alumno vaya superando metas y necesite cada vez actuaciones menos individualizadas. Al adaptar, debemos atenernos siempre a los criterios de realidad y éxito.

- Considerar la realización de adaptaciones curriculares como un proceso dinámico y flexible, por lo tanto, sometido a modificaciones continuas.

- Siempre es preferible reflejar las decisiones por escrito, identificando, al mismo tiempo, las personas implicadas y su grado de responsabilidad.

\section{Estrategias para la aplicación de las Adaptaciones curriculares de pequeño porte o no significativas.}

En las escuelas brasileñas hay dos formas de organizar el personal docente, de forma que en las primeras series el profesor regente de aula es el que imparte la mayoría de las áreas, mientras que de quinta serie en adelante las enseñanzas son organizas por profesores especialistas para las diferentes áreas.

Como para nuestra propuesta lo que pretendemos es que se responsabilice de las DACI 
aquel profesor que imparte el área ya sea de las primeras series o de quinta en adelante siempre utilizaremos este término para evitar equívocos.

Planificación de recursos humanos

- Profesorado de apoyo o especialista en educación especial.

- El desdoble de grupos

- Apoyos del profesorado del nivel paralelo o de otros profesores del centro.

Planificación de recursos materiales

- Uso del ordenador

- El libro didáctico y los materiales complementarios

- Planificación del uso de espacios

Planificación de tiempos

- Momentos programados con actividades en las que la mayoría de los alumnos pueden hacer sus trabajos de forma autónoma.

- Explicaciones directas del profesorado al grupo alternadas con explicaciones individualizadas.

Metodología

- Crear autonomía del grupo en cuanto a la búsqueda y consecución de sus aprendizajes.

- Programación de actividades con diferentes niveles de profundización.

- Alternancia de estrategias de trabajo cooperativo y participativo donde se de cabida a la individualidad,

- Técnicas de monitorización por parte de unos alumnos a otros alumnos.

- Trabajo en equipo.

- Metodologías globalizadoras

\section{Responsables de la elaboración de las adaptaciones curriculares significativas}

La elaboración y desarrollo de adaptaciones curriculares es un proceso compartido de toma de decisiones que afecta al Proyecto Político Pedagógico, a las programaciones didácticas y a las programaciones de aula, puesto que se trata de modificar la ayuda educativa y didáctica diseñada con carácter general para todo el alumnado.

El hecho de ser un proceso compartido viene condicionado por las funciones que la normativa atribuye, en cuanto a su planificación y aplicación, al profesor responsable de clase y el resto de los profesores. Por lo común, las decisiones de carácter general recogidas en la DACI (áreas 
cursadas, horario, criterios de promoción, colaboración con la familia...) serán responsabilidad de los órganos gestores del centro escolar coordinadas por el profesor responsable de clase, que es quién conoce al alumno, con el asesoramiento del profesorado de apoyo, de los servicios de orientación, de aquellos servicios de apoyo con los que cuente la escuela,... a partir de la información obtenida de los profesores del alumno y del informe diagnóstico.

La programación de las distintas áreas y materias que cursa el alumno corresponde a los distintos profesores, que podría contar para ello con la colaboración de los servicios de apoyo. En algunas áreas de carácter más instrumental (lengua, matemáticas) la participación de los profesores de apoyo, suele resultar muy relevante.

La aplicación de estas adaptaciones corresponde a los profesores de área, con la participación intensa de los profesores de apoyo (si los hubiera) en el caso de que se prevean medidas de apoyo.

\section{Proceso de elaboración de una adaptación curricular}

Para ello proponemos una serie de fases:

$1^{\text {a }}$ Fase:

Delimitación de los profesionales implicados, que por lo general suelen ser el profesor responsable de clase o tutor, el profesor de apoyo, los profesores especialistas de cada una de las áreas curriculares implicadas y el orientador o coordinador.

$2^{\mathrm{a}}$ Fase:

Delimitación de las necesidades educativas, que pueden ser pedagógicas, que correspondería a los profesores de apoyo; o curriculares, que corresponderían al profesor responsable de clase y los profesores que le imparten docencia en cada una de las áreas curriculares.

$3^{\text {a }}$ Fase:

Integración de datos recogidos en la evaluación diagnóstica previa y que daría paso a la elaboración del Informe previo y la determinación de la respuesta educativa adecuada. $4^{\mathrm{a}}$ Fase:

Se produciría la implementación del DACI, es decir, su puesta en práctica. $5^{\text {a }}$ Fase:

Reflejaría las propuestas para las sucesivas coordinaciones y seguimientos. $6^{\mathrm{a}}$ fase:

Culminaría con la evaluación (valoración global y específica), con las posibles propuestas de 
modificaciones y la revisión y evaluación del plan propuesto inicialmente.

\section{MODELO DACI}

Adaptaciones curriculares no significativas.

\begin{tabular}{|c|c|}
\hline \multicolumn{2}{|c|}{ DATOS PERSONALES } \\
\hline \multicolumn{2}{|c|}{$\begin{array}{l}\text { NOMBRE Y APELLIDOS DEL ALUMNO: } \\
\text { FECHA DE NACIMIENTO: } \\
\text { ESCOLARIZADO EN: } \\
\text { CICLO }\end{array}$} \\
\hline \multicolumn{2}{|c|}{$\begin{array}{c}\text { INFORMACION PREVIA DEL ALUMNO } \\
\text { Escolarización previa, datos escolares de relevancia, informes de años anteriores, resultados evaluación,... }\end{array}$} \\
\hline \multicolumn{2}{|c|}{$\begin{array}{l}\text { NECESIDADES EDUCATIVAS } \\
\text { Señalará en qué ámbitos y qué respuesta educativa se precisa: } \\
\text { (adaptación de elementos y/o provisión de medios de acceso). }\end{array}$} \\
\hline \multicolumn{2}{|c|}{ ASPECTOS QUE SE MODIFICAN } \\
\hline \multicolumn{2}{|c|}{ INFORMACIÓN RELEVANTE PARA LA TOMA DE DECISIONES } \\
\hline ASPECTOS POSITIVOS & ASPECTOS NF.FATIVOS \\
\hline
\end{tabular}




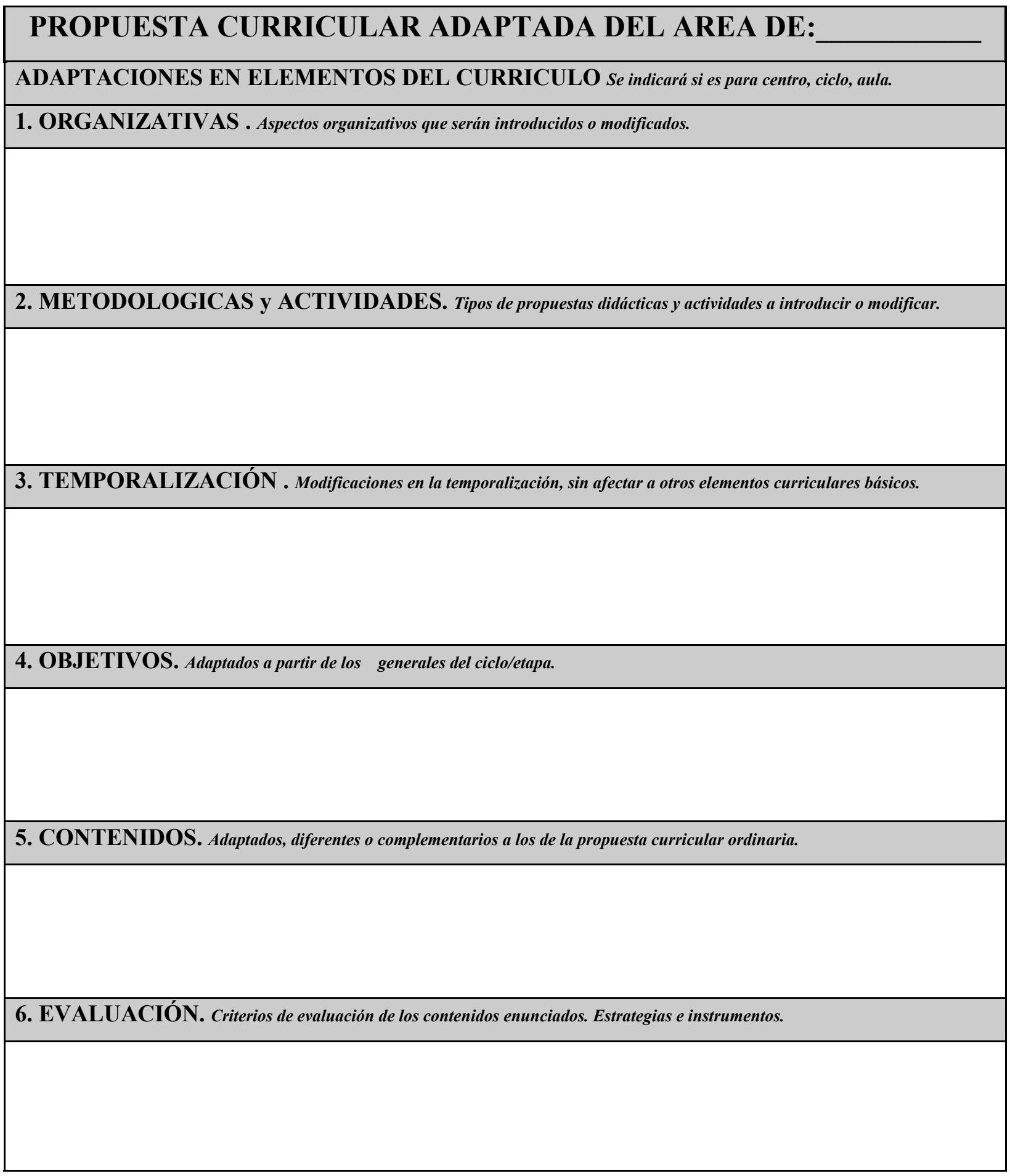




\section{OTRAS DECISIONES}

\section{OTRAS MEDIDAS. Aula especifica, periodo opuesto.}

APOYOS. Modalidad, temporalización, profesionales implicados,...

INTERVENCIÓN SOCIO-FAMILIAR. Propuesta de colaboración de la familia y/u otras entidades en relación a los objetivos que se plantean

SEGUIMIENTO. Temporalización, profesionales implicados, decisiones,,...

EVALUACIÓN DE LA ACI.

PROFESIONALES QUE HAN PARTICIPADO EN LA ELABORACIÓN DE LA ACI

\begin{tabular}{|l|l|l|}
\hline \multicolumn{2}{|l|}{ Nombre } & Cargo \\
\hline \multicolumn{2}{|l|}{ FECHA DE } & \\
ELABORACIÓN DEL & DURACIÓN & \\
DACI & PREVISTA & \\
\hline & & FECHAS DE REVISIÓN \\
\hline
\end{tabular}

RESPONSABLE DEL DACI : 


\section{BIBLIOGRAFÍA}

- Blanco, R (Coord). (1996) Alumnos con Necesidades Educativas Especiales y Adaptaciones Curriculares. Equipo de trabajo del centro de Desarrollo Curricular. MEC. Madrid.

- Bolivar, A. (2000) Los centros educativos como organizaciones que aprenden. Madrid: La Muralla

- Casanova. M.A. (2006): Diseño curricular e innovación educativa. Madrid: La Muralla

- Coll, C. (1987): Psicología y Currículum. Una aproximación psicopedagógica al currículum escolar. Barcelona, Laia.

- Delors, J. (1996) La educación encierra un tesoro. Madrid: Santillana

- Galve , J.L., Trallero, M, Sebastián, E. y otros (2002) ACI. Las adaptaciones curriculares individuales, Madrid: CEPE.

- Gimeno, S. (2005): La educación que aún es posible. Madrid, Ed. Morata.

- $\quad$ MEC (1996) Ley de Directrices y Bases, de Educación Brasileña, Ley 9394 de 20 de diciembre de 1996. Brasilia: MEC

- MEC (1999) Parâmetros curriculares nacionais. Adapaçoes curriculares. Brasilia. MEC-SEF-SEESP

- Procuraduría dos Directos do Cidadao (2004) O accesso de alunos com defiência as escolas e classes comuns da rede regular. Barasilia: PDC

- Sebastián, E. Galve, J.L., Ayala, C.L., Cabrera, J. (1996) PDC: Programas de Diversificación Curricular. Madrid: CEPE.

- Sebastián Heredero, E. (1999) NEE y Adaptaciones curriculares. Marilia (SP) UNESP-FFC- Dto. de educación especial.

- Sebastián Heredero, E. (2007) Necesidades educativas especiales y adaptaciones curriculares. Araraquara (SP): UNESP-FCLAR

- Sebastián Heredero, E. (2007) La atención a la diversidad en el nuevo contexto de la sociedad plural. En Sebastián, E. y Martin, M. (Coord) (2007) Educación y sociedad global: demandas y aportaciones. Alcala de Henares (Madrid): Servicio de publicaciones de la UAH

- Secretaria da Educação Especial. (2006) Inclusão: um desafio para os sistemas de ensino. Brasilia: MEC

- Secretaria da Educação Especial. (2000) Escola Viva. Garantindo o acesso e permanência de todos os alunos ne escola. Alunos com necessidade educacionais especiais. Brasilia: MEC

- Warnock, M. (1981). Meeting Special Education Needs. Londres. Her Majesty's Stationary Office, Government Bookshops (sixt impression) 